\begin{tabular}{|c|c|}
\hline Title: & $\begin{array}{l}\text { New FPGA-based and inline-capable measuring method for the identification of magnetic } \\
\text { losses in electrical steel }\end{array}$ \\
\hline Authors: & Marc Veigel, Patrick Winzer, Jan Richter, Martin Doppelbauer \\
\hline Institute: & $\begin{array}{l}\text { Karlsruhe Institute of Technology (KIT) } \\
\text { Elektrotechnisches Institut (ETI) } \\
\text { Hybrid Electric Vehicles (HEV) }\end{array}$ \\
\hline Type: & Conference Proceedings \\
\hline Published at: & $\begin{array}{l}2015 \text { 5th International Electric Drives Production Conference (EDPC), Nuremberg, } 2015 \\
\text { Publisher: IEEE, Piscataway (NJ) } \\
\text { Year: } 2015 \\
\text { ISBN: } 978-1-4673-7512-2 \\
\text { Pages: } 1-6\end{array}$ \\
\hline Hyperlinks: & DOI: https://doi.org/10.1109/EDPC.2015.7323200 \\
\hline
\end{tabular}

(C) 2015 IEEE. Personal use of this material is permitted. Permission from IEEE must be obtained for all other uses, in any current or future media, including reprinting/republishing this material for advertising or promotional purposes, creating new collective works, for resale or redistribution to servers or lists, or reuse of any copyrighted component of this work in other works. 


\title{
New FPGA-Based and Inline-Capable Measuring Method for the Identification of Magnetic Losses in Electrical Steel
}

\author{
Marc Veigel, Patrick Winzer, Jan Richter, Martin Doppelbauer \\ Karlsruhe Institute of Technology (KIT) \\ Elektrotechnisches Institut (ETI) - Hybrid Electric Vehicles \\ Karlsruhe, Germany \\ marc.veigel@kit.edu
}

\begin{abstract}
The identification and characterization of magnetic losses in electrical steel is the basis for the improvement of current drive technologies and quality assurance in the manufacturing processes. The enormous demands on drives regarding high frequencies, non-sinusoidal waveforms and polarizations in the area of the saturation flux density yield increased requirements for suitable testing devices. Thus, it is necessary to develop a new measuring system that combines the requirements of series production and measurement precision. As a second step it is possible to establish a measurement strategy that differ and separate the influence on the magnetic losses of every single production step inside the manufacturing process. For this application a fast, flexible and high performant modular measuring device is built up and is described in this paper.
\end{abstract}

Keywords—magnetic losses; materials testing; magnetic analysis; measurment; production engineering

\section{INTRODUCTION}

Present commercial testing devices for electrical steel that are conform to international standards provide measurements under sinusoidal waveform conditions .The frequency ranges from a few $\mathrm{Hz}$ to about $20 \mathrm{kHz}$ and the flux density amplitude from $0.1 \mathrm{~T}$ to $2 \mathrm{~T}$ depending on the geometry of the specimen the tester and the material [1], [2]. The control times are strictly linked to the requested operation point and amount values from at least $10 \mathrm{~s}$ to a couple of minutes [3]. In total the identification of core losses in the whole working area of the drive takes a few hours [3].

Standardized measurements cover the investigation of single sheets, toroidal cores and Epstein strips with variable dimensions [4], [5], [6], [7]. Although the geometry of the specimen and the particular distribution of the flux density can have a significant effect on the measuring results the geometry of these standardized test samples is generally far away from the actual target geometry. Furthermore increasing quantities force the drive production industry to reconsider the manufacturing processes leading to different production impacts to the laminated sheet package but also on the material itself [8], [9]. These impacts generally are significant in the appearance of core losses especially in eddy currents and are also not possible to identify with the standardized test equipment.
With the individual adaptable measuring system that is presented in this paper it is possible to analyze the mentioned standardized test specimen but also custom-designed geometries like single teeth which can directly be further processed to a stator's laminated core. The principles of operation of the measuring device are illustrated by the example of the determination of the magnetic losses in a custom single tooth geometry but can easily be transferred to the standardized measurements. However, the measurement device can also be used for standardized measurements.

The theoretical fundamentals and measuring conditions for the determination of magnetic losses in standardized specimen are well-defined in industrial standards [4], [5], [6], [7]. It is necessary to compose a magnetic circuit with at least one primary winding for the magnetization of the material and one secondary winding to determine the resulting magnetic flux. According to the single tooth geometry of the specimen an adapted and suitable high performable yoke has been designed and wound with a primary winding to establish the magnetic circuit. The secondary winding is also placed in the area of the yoke using a plastic support geometry. This arrangement avoids time-consuming and complex winding processes for every single test object as it is usual for toroidal cores and enables inline capability of the measurement device during series production.

The measuring adapter is powered by a high performant linear amplifier that consists of multiple amplifier modules of which a single one can deliver up to $50 \mathrm{~A}$ peak at $60 \mathrm{~V}$. The amplifier modules are connected in parallel to increase the possible output current in order to achieve high values of magnetic field strength in the specimen. The amplifier modules are parallelized using small series shunts and an FPGA based high speed control algorithm to maintain equal output current for each module. The output stage is implemented in class $\mathrm{H}$ topology to ensure safe operation of the output transistors as well as lower losses and therefore lower temperature loads compared to conventional topologies.

With regard to cost of material and weight saving aspects in mobile drive applications of today the magnetic behavior of the material in high saturated flux density areas gains more and more significance. In this highly nonlinear area of magnetization a fast and adaptive flux controller is indispensable to provide the 


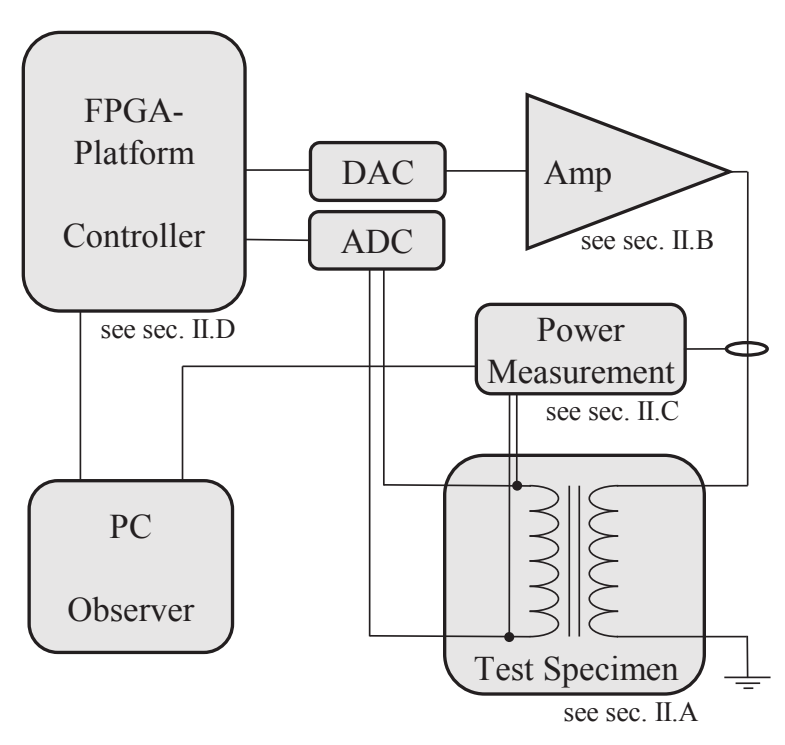

Fig. 1 Schematic view of the measurement system

sinusoidal shape of the polarization waveform required by the industrial standards. Because of this reason the FPGA-based hardware platform is used to provide a maximum controllable fundamental wave of $20 \mathrm{kHz}$ and similar control times of a few seconds in every operation point from $0.1-2 \mathrm{~T}$.

As opposed to other control techniques the control algorithm is implemented in the time domain and not in frequency domain and runs directly in the FPGA program [3]. This is why timeconsuming tasks of calculation like fourier transformations or slow data transfers to a PC are not needed in the control loop and small control times are achieved enabling inline capability.

\section{MEASUREMENT SYSTEM}

The general principles of operation of the testing device are presented in this section. It should be noted that every single subsystem that is shown in Fig. 1 is explained in separate sections. The task that has to be performed is to set well defined boundary conditions inside of an electromagnetic circuit (II.A) and to perform a proper power measurement and signal capturing afterwards (II.C). Because there is no sufficient information about the materials behavior a controlled loop (II.D) is necessary to meet the requested conditions in every operation point. The controllers output has to be amplified to supply the circuit's windings with adequate power. This task also comes along with great demands on hard- and software development to meet the requirements and is introduced in chapter II.B .

\section{A. Electromagnetic circuit}

The description of the electromagnetic circuit of standardized measurements is already done in the specific industry standard. In this section a new kind of measurement setup is introduced that enables the characterization of a semiprocessed geometry in the form of a single tooth geometry.

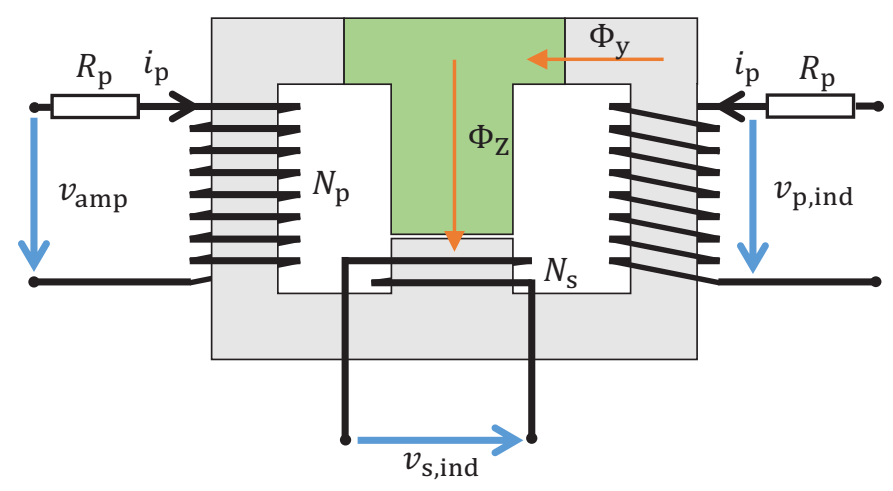

Fig. 2 Magnetic circuit of the single tooth tester and declaration of tooth flux $\phi_{\mathrm{Z}}$ and yoke flux $\phi_{\mathrm{y}}$

To set up the system equations for the controller the magnetic circuit of the testing device has to be considered and described. As shown in Fig. 2 the excitation of the whole system is an equal and controllable amplifier voltage powering both primary windings $v_{\text {amp }}(t)$. The equality is forced by the parallel connection to the output modules. This voltage is decreased by the ohmic voltage drop at the wire resistor of each winding system $R_{\mathrm{p}}$ and leads to the voltage equation of the primary windings

$$
v_{\mathrm{amp}}(t)=R_{\mathrm{p}} \cdot i_{\mathrm{p}}(t)+v_{\mathrm{p}, \text { ind }}(t)
$$

where

$$
\begin{array}{lll}
v_{\text {amp }}(t) & : & \text { amplifier voltage; } \\
R_{\mathrm{p}} & : & \text { winding resistor; } \\
i_{\mathrm{p}}(t) & : & \text { winding current; } \\
v_{\mathrm{p}, \text { ind }}(t) & : & \text { primary induced voltage; }
\end{array}
$$

The induced voltage $v_{\text {p,ind }}(t)$ is caused by the alteration of the magnetic flux inside of the winding

$$
v_{\text {p,ind }}(t)=N_{\text {prim }} \cdot \frac{\mathrm{d} \Phi_{\mathrm{y}}(t)}{\mathrm{d} t}
$$

where

$$
\begin{array}{lll}
N_{\mathrm{p}} & : & \text { Turns of primary winding; } \\
\Phi_{\mathrm{y}}(t) & : & \text { magnetic flux in corresponding } \\
& \text { part of yoke; }
\end{array}
$$

The division of the magnetic flux into the left and right part of the yoke will be symmetric due to the same amplifier voltage supplying them. Only a different voltage drop at the windings resistor eventually caused by different air gap condition will cause negligible differences in the induced voltage. Therefore a relationship between primary and secondary induced voltage can be established. The only condition is a negligible path of magnetic flux leakage what is provable with FEM simulation tools.

$$
\begin{gathered}
v_{\mathrm{s}, \text { ind }}(t)=N_{\mathrm{s}} \cdot \frac{d \Phi_{Z}(t)}{d t}=N_{\mathrm{s}} \cdot \frac{2 \cdot \mathrm{d} \Phi_{\mathrm{y}}(t)}{\mathrm{d} t} \\
v_{\mathrm{s}, \text { ind }}(t)=2 \cdot \frac{N_{\mathrm{s}}}{N_{\mathrm{p}}} \cdot u_{\mathrm{p}, \text { ind }}(t)
\end{gathered}
$$

where 


$$
\begin{array}{lll}
N_{\mathrm{S}} & : & \text { turns of secondary winding; } \\
\Phi_{\mathrm{Z}}(t) & : & \text { magnetic flux in tooth; }
\end{array}
$$

This transformer equation is always valid even in the case of nonlinear magnetics caused by saturation. Fig. 2 illustrates the electromagnetic conditions up to this point.

\section{B. Amplifier System}

The measuring adapter is powered by a high performance linear amplifier that consists of multiple parallel amplifier modules, one of which is depicted in Fig. 3. A single amplifier module can deliver up to $50 \mathrm{~A}$ peak at $60 \mathrm{~V}$. Its bandwidth exceeds the $100 \mathrm{kHz}$ range and the slew rate is greater than $100 \mathrm{~V} / \mu \mathrm{s}$.

The output stage of the amplifier modules is implemented in class $\mathrm{H}$ topology to ensure safe operation of the output transistors as well as lower losses and therefore lower temperature loads compared to conventional topologies. The parallelization of the amplifier modules allows linear scaling of the output current and thus, the output power of the amplifier system. It is implemented using small series shunt resistors and an FPGA based high speed control algorithm to maintain equal output current for each module. The algorithm calculates individual input voltages for the amplifier modules, which are supplied by a 16bit digital-analog-converter.

\section{Power Measurement}

The power that is absorbed by this electromagnetic system is a composition of the ohmic losses in the primary windings, the core losses in the yoke and the core losses in the test specimen which are to be determined. By multiplying equation (1) with the primary current and replacing the induced voltage by the measured secondary voltage using equation (2) the systems power equation is received
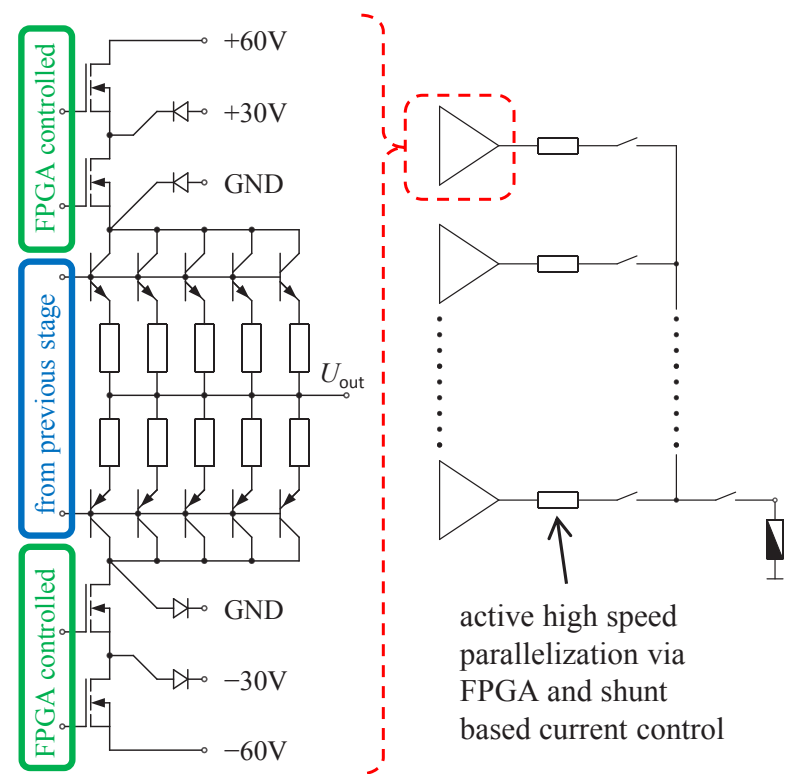

Fig. 3 Output stage of an amplifier module and parallelization of amplifier modules

$$
\underbrace{v_{\mathrm{amp}}(t) \cdot i_{\mathrm{p}}(t)}_{A}=\underbrace{R_{\mathrm{p}} \cdot i_{\mathrm{p}}{ }^{2}(t)}_{B}+\underbrace{\frac{N_{\mathrm{s}}}{2 \cdot N_{\mathrm{p}}} \cdot v_{\mathrm{s}, \text { ind }}(t) \cdot i_{\mathrm{p}}(t)}_{C}
$$

where

$$
\begin{array}{lll}
A & : & \text { system power; } \\
\text { B } & : & \text { ohmic losses in winding; } \\
C & : & \text { core magnetization and losses; }
\end{array}
$$

This relationship shows the possibility to determine the system's core magnetization and losses by observing the secondary voltage and the primary current over time. This oscillating waveform can be split up into a part of reactive power that it equal to the magnetization of the core and a part of active power that is equal to the core's losses that have to be determined. For an adequate power identification also higher harmonics of the waveform has to be taken into account and a suitable sampling frequency needs to be chosen.

Due to the yoke arrangement the identified core losses are also influenced by the yoke's core losses itself even though the saturation flux density is much higher and the coercivity is much lower than those of the specimen. However, the magnetizing conditions inside of the yoke's arrangement are not changing by putting in another tooth specimen. A higher magnetic resistance of the magnetic circuit that can be caused by worse quality of the tooth or other air gap conditions results in the demand of a higher magnetic tension to drive the requested flux and therefore a higher current in the windings but the losses of the yoke only depend on the flux waveform $\Phi_{\mathrm{y}}(t)$. Thus, the yoke's flux and the yoke's losses are clearly defined by the operation point and not by the quality of the specimen. These conditions guarantee comparable measurement results taking the yoke's losses into account.

\section{Controlling}

In order for the power loss measurement of the test specimen to be precise the secondary induced voltage $v_{\mathrm{s} \text {,ind }}$ has to be controlled to a sinusoidal waveform with a total harmonic content as low as possible. Due to iron saturation a nonsinusoidal winding current $i_{\mathrm{p}}$ has to be fed to the primary winding to reach that goal. This is achieved by supplying a nonsinusoidal amplifier voltage $v_{\mathrm{amp}}$. Thus, the relation between the secondary induced voltage $v_{\text {s,ind }}$ and the amplifier voltage $v_{\text {amp }}$ is strongly nonlinear due to the ohmic winding resistance. This makes control of the system a challenging task.

Since all electric and magnetic quantities are periodical the principal of repetitive control can be applied. This was already applied to position and speed control in hard disc [8] or optical disk drives [9]. Recently the same principle was used to mitigate current harmonics in permanent magnet synchronous machines [10].

It is based on the idea that an error in the secondary induced voltage $v_{\text {s,ind }}$ can be identified by measurement and be converted to an additional voltage demand of the amplifier voltage $v_{\text {amp }}$ when the phase is identical again. This approach 


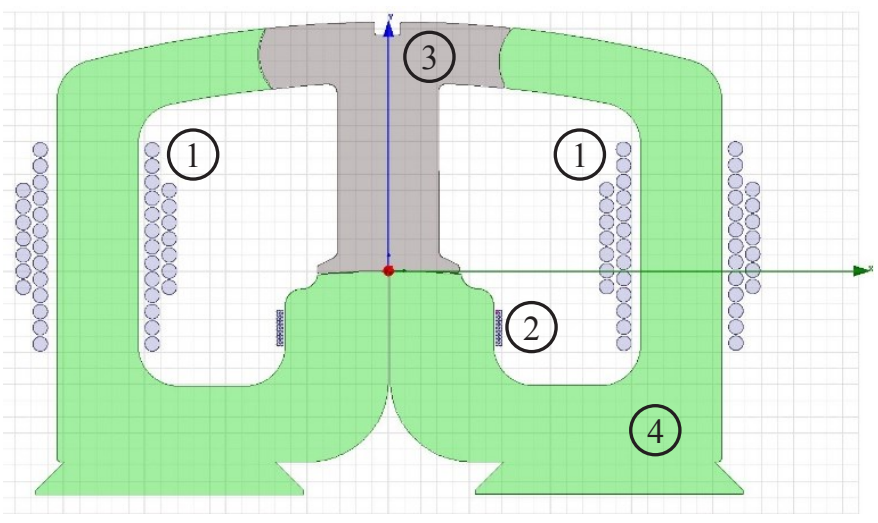

Fig. 4 Yoke geometry and position of primary and secondary winding system. (1) Primary windings (2) Secondary winding (3) Tooth specimen (4) yoke

yields a controller that converges within several electrical periods.

The controller is implemented on a field programmable gate array (FPGA) to realize fast sample rates and control times needed for quality assurance in series production in end of line testing. Depending on the test specimen, the requested frequency and the polarization total control and measurement times between one and five seconds for each operation point are achieved. The form factor of the secondary induced voltage $v_{\mathrm{s}, \text { ind }}$ is monitored and a power measurement is not triggered before the requirements of [5] are strictly kept. An example of the dynamic control performance is given in section IV.

\section{EXPERIMENTAL SETUP}

An overview of the performance data of the built up test bench described in section II is given in this chapter. Starting with the electromagnetic circuit the optimum numbers of turns of the secondary and primary windings $N_{\mathrm{p}}$ and $N_{\mathrm{s}}$ need to be figured out by paying attention to the amplifiers data. The maximum output current depends on the number of modules that are in use and can be scaled up to 400 A using 8 modules in parallel. On
TABLE I PERFORMANCE DATA OF EXAMPLE SETUP

\begin{tabular}{|l|c|}
\hline$f_{\max }$ & $2 \mathrm{kHz}$ \\
\hline$B_{\max }$ & $1.8 \mathrm{~T}$ \\
\hline$I_{\mathrm{p}, \max }$ & $400 \mathrm{~A}$ \\
\hline$V_{\mathrm{p}, \max }$ & $60 \mathrm{~V}$ \\
\hline$V_{\mathrm{s}, \max }$ & $80 \mathrm{~V}$ \\
\hline$N_{\mathrm{p}}$ & $5 / 10 / 20$ \\
\hline$N_{\mathrm{s}}$ & $5 / 10 / 20$ \\
\hline
\end{tabular}

the other hand the maximum output voltage is limited to an amplitude of $60 \mathrm{~V}$. These boundary conditions the parameters of the electromagnetic circuit and the requested measuring range up to $2 \mathrm{kHz}$ and $1.8 \mathrm{~T}$ lead to a scalable winding system containing $4 \times 5$ primary and secondary windings that can automatically set to parallel or serial during the measurement process by using an external switching relay circuit. With this technique an independent number of turns can be set to 5,10 or 20 during the measurement. The maximum secondary input voltage reaches $80 \mathrm{~V}$ of amplitude and is scaled up or down to the Analog-Digital-Converters input range of $10 \mathrm{~V}$ with a scalable operational amplifier circuit as well for an optimum resolution in the values range. Table I shows the maximum ratings of the previous described setup. Of course, higher values in frequency and flux density can be reached by choosing other winding turn numbers.

The yoke geometry is composed of a cobalt iron lamination that reaches the saturation induction at $2.3 \mathrm{~T}$ and a coercitivity lower than $0.4 \mathrm{~A} / \mathrm{cm}$. The sheet thickness is set to $100 \mu \mathrm{m}$ to minimize the eddy current losses of the adhered lamination sheets. Furthermore the cross section areas of the yoke are increased in comparison to the tooth to decrease the flux density and to minimize the losses that are generated outside of the specimen. A profile of the measuring arrangement is depicted in Fig. 4.
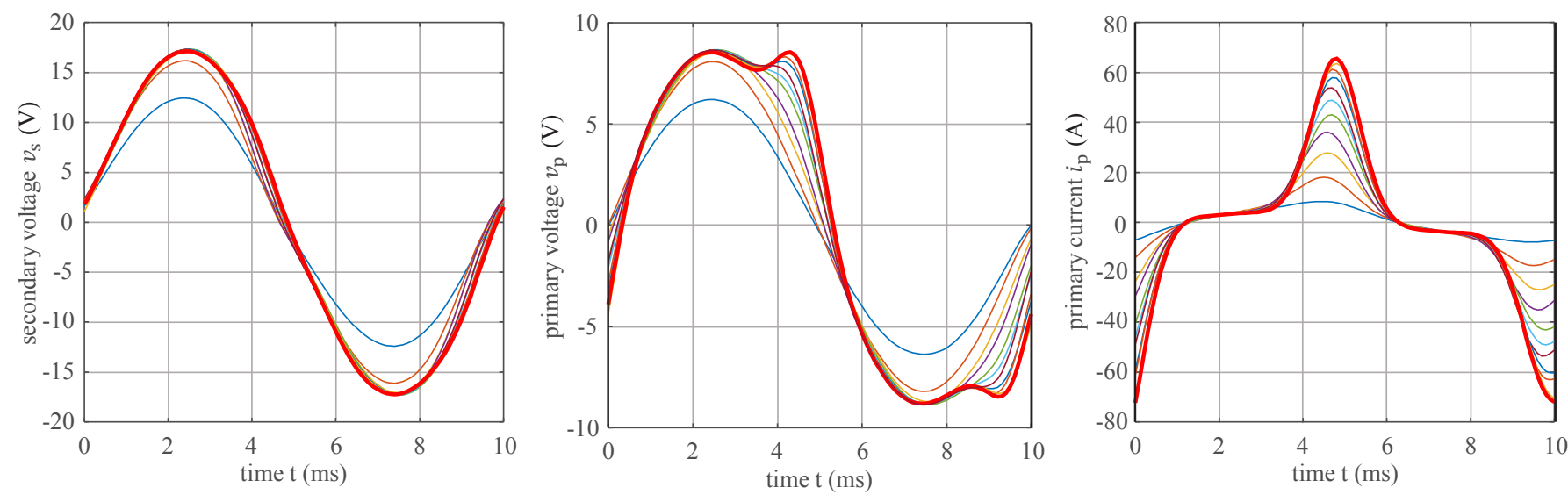

$-0.06 \mathrm{~s}-0.12 \mathrm{~s}-0.18 \mathrm{~s}$

$0.24 \mathrm{~s}$

$-0.3 \mathrm{~s}$

$0.36 \mathrm{~s}-0.42 \mathrm{~s}$

$0.48 \mathrm{~s}$

0.54

$0.6 \mathrm{~s}-0.66 \mathrm{~s}$

Fig. 5 Temporal evolvement of the system parameters at an operation point of $100 \mathrm{~Hz}$ and $1.5 \mathrm{~T}$. Every single color describes one iteration of the control algorithm and takes 6 times the individual period length of the fundamental wave. In this case the final waveform of the primary voltage which is indicated by the red line is reached after 11 iterations and therefore 0.66 seconds. The dashed line in the first diagram shows the sinusoidal reference voltage waveform. 
The FPGA hardware platform is used for the control algorithm of the module currents and flux density. All system values that are necessary for controlling module currents or the primary voltage are sampled synchronously leading to a true input sampling rate of $1.5 \mathrm{MHz}$ and a system clock rate of 120 $\mathrm{MHz}$ allowing a fast and accurate flux density control. The number of samples per period is set to 1000 to give the controller the opportunity to take precisely influence on higher harmonics of the primary voltage.

The independent power measurement system is represented by a Yokogawa WT3000 Precision Power Analyzer with a wide frequency input range of up to $1 \mathrm{MHz}$ and directly implemented features like the analysis of higher harmonics or THD calculations [10]. The current measurement is implemented by Closed-Loop Transducers of type LEM IT 200-S ULTRASTAB which provide an accuracy of $0.0084 \%$. Furthermore suitable input current ranges are realized by different numbers of turns around the Closed-Loop Transducers to further increase the measurement accuracy. The overall system including the FPGA platform and the power measurement is operated by a $\mathrm{PC}$ via USB. The software LabView from National Instruments is used as Front End, allowing the user manual configurations or to run fully automated series of measurement.

\section{RESULTS}

The results that are achieved with the measurement system including the behavior of the controller and the power measurement system are presented in this section. Starting with the behavior of the control algorithm the temporal evolvement of the most relevant system parameters are shown in Fig. 5. The waveforms are recorded directly from the measurement system by reading the appropriate memory in the FPGA. In this case the controller needs eleven iterations to reach the exit condition that is defined via a minimum value of the error signal and a requested form factor of the secondary voltage that is claimed

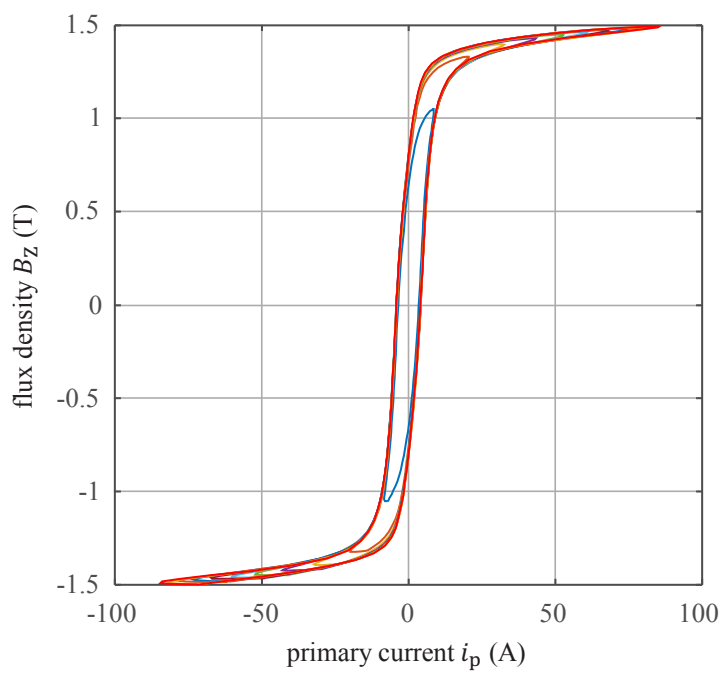

Fig. 6 Temporal evolvement of the hysteresis loop depending from primary current and flux density at $100 \mathrm{~Hz}$ and 1.5 Tesla.
TABLE II EXIT CONDITIONS FOR CONTROL ALGORITHM

\begin{tabular}{|c|c|c|c|}
\hline Iteration & Time(s) & Error (\%) & Form factor \\
\hline 1 & 0.06 & 50 & 1.1210 \\
\hline 3 & 0.18 & 10 & 1.1395 \\
\hline 5 & 0.30 & 5 & 1.1441 \\
\hline 7 & 0.42 & 2 & 1.1352 \\
\hline 9 & 0.54 & $<1$ & 1.1249 \\
\hline 11 & 0.66 & $<1$ & 1.1189 \\
\hline
\end{tabular}

by [5]. The error signal is defined by the difference between the sinusoidal reference signal and the measured signal of the secondary voltage.

Table II indicates the sequence of the controller iterations. When both exit conditions are fulfilled the algorithm stops and enables the power determination. The qualitative progression of the hysteresis loop can be calculated via integration of the secondary voltage signal and the primary current which is proportional to the magnetic field strength and is shown in Fig. 6. The conditions are the same as in Fig. 5.

A direct calculation of the magnetic field strength that drives the magnetic flux inside of the tooth geometry is strongly influenced by unpredictable quantities like additional air gaps which are probably caused by production tolerances and inhomogeneous flux distribution and is therefore not shown. However, the comparability of the measurements is guaranteed by a clearly defined flux density inside the single tooth that does not change with another type of specimen.

If the control algorithm gives the approval for power measurement the internal calculated power dissipations will be read from the Yokogawa Power Analyzer and saved into the measurements protocol. An example is given in Fig. 7 where an

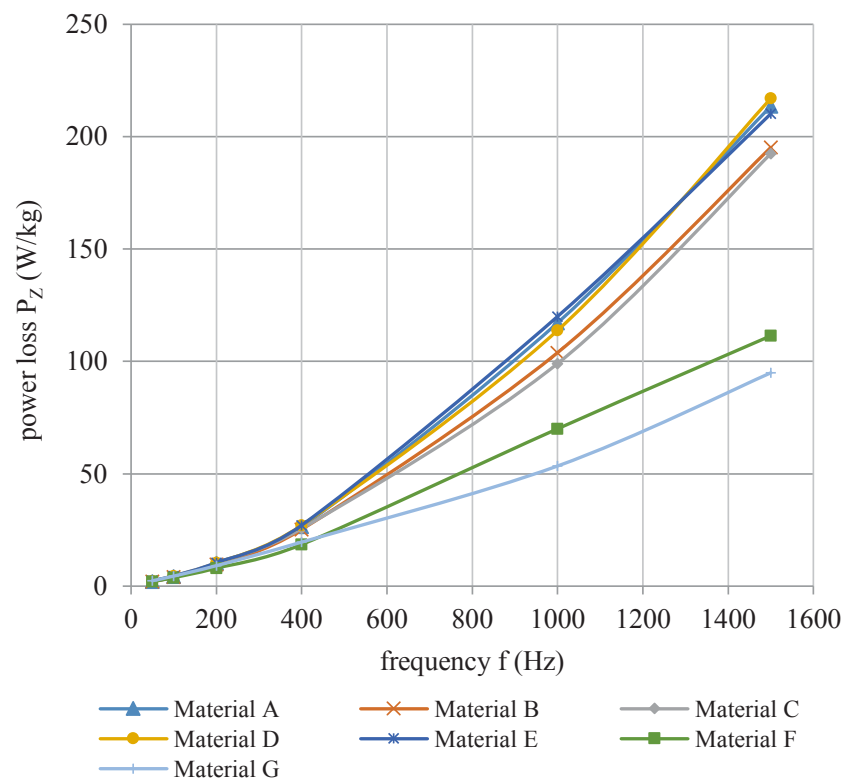

Fig. 7 Effect of different materials on the magnetic losses over frequency in a single tooth geometry under controlled sinusoidal magnetizing conditions with an amplitude of $1 \mathrm{~T}$. 
investigation of the influence of the usage of different materials on the magnetic losses is indicated. In this series of measurements different suppliers of a M330-35A material quality (Material A - Material E) were compared with a NO20 quality (Material F) and a NO10 quality (Material G). The whole series that consists of 42 operation points was finished within 10 minutes including the change of the teeth to underline the inline capability of the measurement system.

\section{CONCLUSION}

A measurement system to identify magnetic losses in electrical steel or in semi-processed geometries like single teeth is presented in this publication. It consists of a digital controller, a power amplifier, a measurement adapter for individual test specimen and a power measurement system. A specifically designed control method allows very short control times so that one operation point can be measured within several seconds even in the presence of strong iron saturation. Thus, the measurement system can be employed as end of line test device in series production for the sake of quality assurance. This enables the analysis of magnetic losses caused by the material and the manufacturing process. The proper functionality is proven by measurements of a single operation point and the frequency dependent power loss identification of seven single teeth. This approach yields a deeper understanding of manufacturing influences on magnetic losses of electrical steel and allows further efficiency improvements in drive technologies.

\section{REFERENCES}

[1] "Measuring Technology for Soft Magnetic Materials," http://www.brockhaus.com, Mar. 2012.
[2] "REMACOMP C for computer controlled measurement of the dynamic characteristics of soft magnetic materials," http://www.magent-physik.de, Apr. 2015.

[3] S. Zurek, P. Marketos, T. Meydan and J. Moses, "Use of novel adaptive digital feedback for magentic measurements under controlled magentizing conditions," IEEE Trans. on Magnetics, vol. 41, no. 11, pp. 4242-4249, 2005.

[4] "Magentic materials - Part 1: Classification," DIN IEC 60404-1, Jun. 2008 .

[5] "Magentic materials - Part 2: Methods of measurement of the magnetic properties of electrical steel strip and sheet by means of an Epstein frame," DIN IEC 60404-2:1996 + A1:2008, Jan. 2009.

[6] "Magentic materials - Part 3: Methods of measurement of the magnetic properties of electrical steel strip and sheet by means of a single sheet tester," DIN IEC 60404-3:1992 + A1:2002 + A2:2009, May 2010.

[7] "Magentic materials - Part 6: Methods of measurement of the magnetic properties of magnetically soft metallic and powder materials at frequencies in the range of $20 \mathrm{~Hz}$ to $200 \mathrm{kHz}$ by the use of ring specimens,“ DIN IEC 60404-6:2003, Oct. 2004.

[8] E. Lamprecht, M. Homme, T. Albrecht "Investigations of eddy current losses in laminated cores due to the impact of various stacking processes," 2nd International Electric Drives Production Conference (EDPC), Nuremberg, Germany, Oct. 2012.

[9] J. Stoll, A. Koelmel, S. Peters, G. Lanza "Flexible production lines for series production of automotive electric drives," 2nd International Electric Drives Production Conference (EDPC), Nuremberg, Germany, Oct. 2012.

[10] M. Tomizuka, T.-C. Tsao, and K.-K. Chew, "Analysis and synthesis of discrete-time repetitive controllers," Journal of Dynamic Systems, Measurement and Control, vol. 111, no. 3, pp. 353-358, Sept. 1989.

[11] J.-H. Moon, M.-N. Lee, and M. J. Chung, "Repetitive control for the track-following servo system of an optical disk drive," IEEE Trans. Control Systems Technology, vol. 6, no. 5, pp. 663-670, Sep. 1998.

[12] J. Richter, T. Lannert, T. Gemassmer, and M. Doppelbauer, "Mitigation of current harmonics in inverter-fed permanent magnet synchronous machines with nonlinear magnetics," in Proc. PCIM Europe 2015, Nuremberg, Germany, May 2015, pp. 725-732.

[13] "Yokogawa WT3000 Precision Power Analyzer," http://www.yokogawa.com, Sep. 2013. 